\title{
Bond Strength of Brackets Bonded with Chemically and Light Activated Materials after Thermal Cycling
}

\author{
Rafael Menezes-Silva ${ }^{*}$, Leonardo Amaral dos Reis ${ }^{2}$, Bhenya Otonni Tostes ${ }^{1}$, \\ Dhelfeson Willyan Douglas de Oliveira ${ }^{3}$, Maria Helena Santos ${ }^{4}$ \\ ${ }^{1}$ Department of Dentistry, Endodontics and Dental Materials, Bauru Dental School, University of São \\ Paulo-USP, Bauru, Brazil \\ ${ }^{2}$ Department of Pathology, Piracicaba Dental School, University of Campinas-UNICAMP, Piracicaba, Brazil \\ ${ }^{3}$ Department of Dentistry, Federal University of Minas Gerais-UFMG, Belo Horizonte, Brazil \\ ${ }^{4}$ Department of Dentistry, Federal University of the "Vales do Jequitinhonha e Mucuri" —UFVJM, Diamantina, \\ Brazil \\ Email: ${ }^{*}$ rafa18ms@hotmail.com
}

Received 28 July 2015; accepted 30 August 2015; published 2 September 2015

Copyright (C) 2015 by authors and Scientific Research Publishing Inc.

This work is licensed under the Creative Commons Attribution International License (CC BY).

http://creativecommons.org/licenses/by/4.0/

(c) (i) Open Access

\section{Abstract}

The aim of this study was to evaluate the bond strength of orthodontic brackets bonded to tooth enamel with different polymer composites after simulated clinical aging, and analyze the enamel/ bond system fracture interface. 80 human premolars, were randomly divided into five groups $(\mathrm{n}=$ 16). G1: CO (Concise Ortodôntica-3M ESPE), G2: SB (SuperBond-Ortho Source), G3: FMO (Fill Magic Ortodôntico-Vigodent), G4: TXT (Transbond XT-3M ESPE) and G5: TP (Transbond Plus Self Etching Primer + Transbond Plus Color Change-3M ESPE). In all groups, the metal orthodontic bracket was bonded to the tooth enamel surface. The specimens were submitted to 3000 thermal cycling baths for $30 \mathrm{~s}$, at temperatures of $5^{\circ} \mathrm{C}, 37^{\circ} \mathrm{C}$ and $55^{\circ} \mathrm{C}\left( \pm 1^{\circ} \mathrm{C}\right)$. Afterwards they were subjected to shear testing and the results were analyzed by the Student's- $t$ test $(p<0.05)$. G1 presented the highest bond strength value, followed by G4 > G5 > G2 > G3. In all groups the majority of the fractures at the interface of the specimens were cohesive, except in G3, which presented the largest number of adhesive fractures. After clinical aging, the chemically activated material (CO) showed higher bond strength than the light activated types (FMO, TXT and TP). The self-etching adhesive system (TP) showed similar bond strength to that of conventional systems (FMO and TXT).

\footnotetext{
${ }^{*}$ Corresponding author.
}

How to cite this paper: Menezes-Silva, R., Reis, L.A., Tostes, B.O., Douglas de Oliveira, D.W. and Santos, M.H. (2015) Bond Strength of Brackets Bonded with Chemically and Light Activated Materials after Thermal Cycling. Advances in Materials Physics and Chemistry, 5, 351-360. http://dx.doi.org/10.4236/ampc.2015.59035 


\section{Keywords}

\section{Dental Bonding, Orthodontic Brackets, Orthodontics}

\section{Introduction}

In the oral cavity, orthodontic brackets are subject to innumerable forces. These may be concentrated in the adhesive layer, or at the adhesive/enamel interface, compromising bracket retention [1] [2]. The bond strength of brackets is influenced by the physical and mechanical properties of the polymer cementing materials, adverse conditions of humidity and temperature present in the mouth, design of the mesh of the bracket base, and method of enamel etching [3]-[7]. In the present study, cementing materials and adhesive systems were evaluated in order to find the best option of material and technique, which would guarantee functional results satisfactory to the patient, without harming the integrity of the tooth.

The progressive technological development of polymer materials associated with acid etching and adhesive systems have provided a wide variety of options of polymer composites on the dental market over the years. For orthodontic bracket bonding there are both chemically and light-activated resins available on the market. Nevertheless, a study evaluating the bond strength of resin composites pointed out a better performance of the chemically activated, in comparison with the light activated types [1].

With so many options on the market, the orthodontist may find it difficult to select and acquire the material that really provides the best properties, and that would be the correct indication for a clinical step in orthodontic correction as important as bracket bonding.

In view of the foregoing, the aim of this study was to evaluate the bond strength of metal orthodontic brackets bonded to tooth enamel with chemically and light-activated materials, and submitted to clinical aging. For this purpose, after cementation and thermal cycling, shear bond strength tests were performed; the structural and elemental characteristics of the surface of materials and enamel/adhesive system/bracket interfaces, as well as the depth of adhesive system penetration into the enamel were analyzed.

\section{Methodology}

The present experiment received approval from the Animal Experimentation Ethics Committee of the Federal University of the Jequitinhonha and Mucuri (Brazil) under registration number 050/09.

A total of eighty first premolars recently extracted due to orthodontic indication were collected, cleaned and stored in distilled water at $6^{\circ} \mathrm{C}$. The selected teeth were examined in order to guarantee that they were free of cracks or other defects in the enamel that could possibly affect the results.

The teeth were randomly divided into five groups $(n=16)$. Each tooth was involved with polyester resin up to the cement-enamel junction. The study groups were identified according to the bracket cementing material. The names, composition and manufacturers of the products used are shown in Chart 1.

After prophylaxis of the vestibular surfaces of the teeth, a metal edgewise bracket, reference 10.30.208 (Morelli, Sorocaba, SP, Brazil), was bonded to the tooth enamel by a same trained operator (RMS), in accordance with the recommendations of each cementing material manufacturer.

In G3, G4 and G5 groups the resin materials were light activated using the LED light appliance (Ultralux EL, Dabi Atlante, Ribeirão Preto, SP, Brazil), at the power from 350 to $500 \mathrm{mw} / \mathrm{cm}^{2}$. In G1 and G2 groups, the resin materials were chemically activated.

Previously to resin cementation, markings were made in the central region of the vestibular surface, by visual method, in order to standardize the position of the brackets in the occlusal-gingival and mesio-distal directions.

All the specimens were stored in distilled water at $37^{\circ} \mathrm{C}$. After 30 days, they were submitted to 3000 thermal cycling baths in a thermal cycling machine (MSCT/3e-ELQUIP, São Paulo, SP, Brazil).Alternately, 30 s-baths were performed for each temperature $\left(5^{\circ} \mathrm{C} \pm 1^{\circ} \mathrm{C}, 37^{\circ} \mathrm{C} \pm 1^{\circ} \mathrm{C}\right.$ and $\left.55^{\circ} \mathrm{C} \pm 1^{\circ} \mathrm{C}\right)$, with an interval of 5 s between each bath.

The mechanical tests were performed in accordance with the ISO/TS 11405 Standard [8] in a universal test machine (EZTest, EZ-L Shimadzu, Japan), using $200 \mathrm{Kgf}$ at $0.5 \mathrm{~mm} / \mathrm{min}$. The maximum loads of bracket/enamel bond rupture were recorded in MPa. 
Chart 1. Chart of identification of groups, characteristics of the materials used for acid etching enamel and cementing brackets, with their commercial brands and respective manufacturers.

\begin{tabular}{|c|c|c|c|}
\hline Group n = 16 & Trademark & Conditioning enamel & Material cementing \\
\hline G1 & $\begin{array}{l}\text { Concise Ortodôntica (CO) } \\
\text { (3 M ESPE) }\end{array}$ & $\begin{array}{c}\text { Phosphoric acid 37\% } \\
\text { (Scotchbond-3 M ESPE) }\end{array}$ & $\begin{array}{l}\text { Chemically actived } \\
\text { cementing agent }\end{array}$ \\
\hline G2 & $\begin{array}{l}\text { SuperBond (SB) } \\
\text { (OrthoSource) }\end{array}$ & $\begin{array}{c}\text { Phosphoric acid 35\% } \\
\text { (Superbond-Ortho Source) }\end{array}$ & $\begin{array}{l}\text { Chemically actived } \\
\text { cementing agent }\end{array}$ \\
\hline G3 & $\begin{array}{l}\text { Fill Magic Ortodôntico } \\
\text { (FMO) (Vigodent) }\end{array}$ & $\begin{array}{l}\text { Phosphoric acid 37\% } \\
\text { (Magic acid-Vigodent) }\end{array}$ & $\begin{array}{l}\text { Light actived } \\
\text { cementing agent }\end{array}$ \\
\hline G4 & Transbond XT (TXT) & $\begin{array}{l}\text { Phosphoric acid 37\% } \\
\text { (Unitek—3 M ESPE) }\end{array}$ & $\begin{array}{l}\text { Light actived } \\
\text { cementing agent }\end{array}$ \\
\hline G5 & $\begin{array}{c}\text { Transbond Plus } \\
\text { Self Etching Primer } \\
+ \\
\text { Trasbond Plus } \\
\text { Color Change (TP) } \\
\text { (3 M) (ESPE) }\end{array}$ & - & $\begin{array}{c}\text { Self-etching cementing agent } \\
+ \\
\text { Light actived } \\
\text { cementing agent }\end{array}$ \\
\hline
\end{tabular}

The interfaces of the specimens were examined under a stereoscopic microscope Stemi 2000C (Carl Zeiss), at 16× magnification, to determine the type of fracture. The fractures were classified as follows [9]: adhesivefracture at the resin/adhesive interface; cohesive in enamel-fracture in the tooth enamel structure; cohesive in resin-fracture in the resin cylinder body; mixed-fracture involving resin and/or adhesive and/or tooth structure.

Test specimens representative of each group were randomly selected and sectioned along their long axis, in the vestibular-lingual direction. The mesial and distal sections were immersed in hydrochloric acid (HCL) (6 mol/l) for $30 \mathrm{~s}$ [10]. Dehydration was performed, using increasing concentrations of acetone. The dehydrated sections were sputter-coated with a thin layer of gold-palladium, and analyzed by a scanning electron microscope (SEM) (CS-3500, Shimadzu, Japan) and a Energy dispersive X-ray spectroscopy (EDS) (CS3200, Oxford, England).

Data analysis was performed with the Statistical Package for Social Sciences (SPSS version 22.0, IBM Inc., USA). Descriptive statistics were performed. The Shapiro-Wilk test and Levene's test were used for the determination of normality and equality of variance, respectively. The results of bracket bond strength were submitted to the One-Way ANOVA with Student's t test as posthoc test. The level of significance was set at $95 \%$ ( $p<$ 0.05).

\section{Results}

The bond strength values of this study are showed in Table 1.

The predominant type of fracture in G1, G2, G4 and G5 groups was cohesive in resin. The G3 group presented the highest number of adhesive fractures. No damage to the enamel surface was observed after debonding, with the exception of two samples from G1, which presented fracture in enamel (Graphic 1).

The SEM photomicrographs images suggested that the largest particles were observed in CO (Figures 1(A)), followed by G2 (Figures 1(B)). The resins FMO (Figure 1(C)), TXT (Figure 1(D)) and TP (Figure 1(E)) were shown to be more homogeneous in the particle size of its filler. From the images it was possible to observe a favorable distribution of inorganic particles in the organic matrix, without the occurrence of agglomerations in the large majority of materials.

$\mathrm{CO} /$ enamel interface showed a thick and uniform hybrid layer along the entire extension of the bracket bonding area with the formation of small tags in the enamel (Figure 1(a)). The SB resin showed a thinner hybrid layer and smaller tags (Figure 1(B) and Figure 1(b)). The hybrid layer formed with the use of FMO (Figure 1(C)) and TXT (Figure 1(D)) shown to be thinner. At higher magnification, the transition zone between the adhesive layer and demineralized enamel of FMO and TXT may be observed in Figure 1(c) and Figure 1(d), respectively. The TP resin showed a thinner hybrid layer and smaller tags, and more continuous, thicker and uniform (Figure 1(E) and Figure 1(e)).

The EDS analysis showed the elements Calcium (Ca) and Silicon (Si) present at the enamel/resin interface in all the spectra. G1 (CO), G3 (FMO), G4 (TXT) and G5 (TP) groups showed the same pattern of spectra in EDS, 

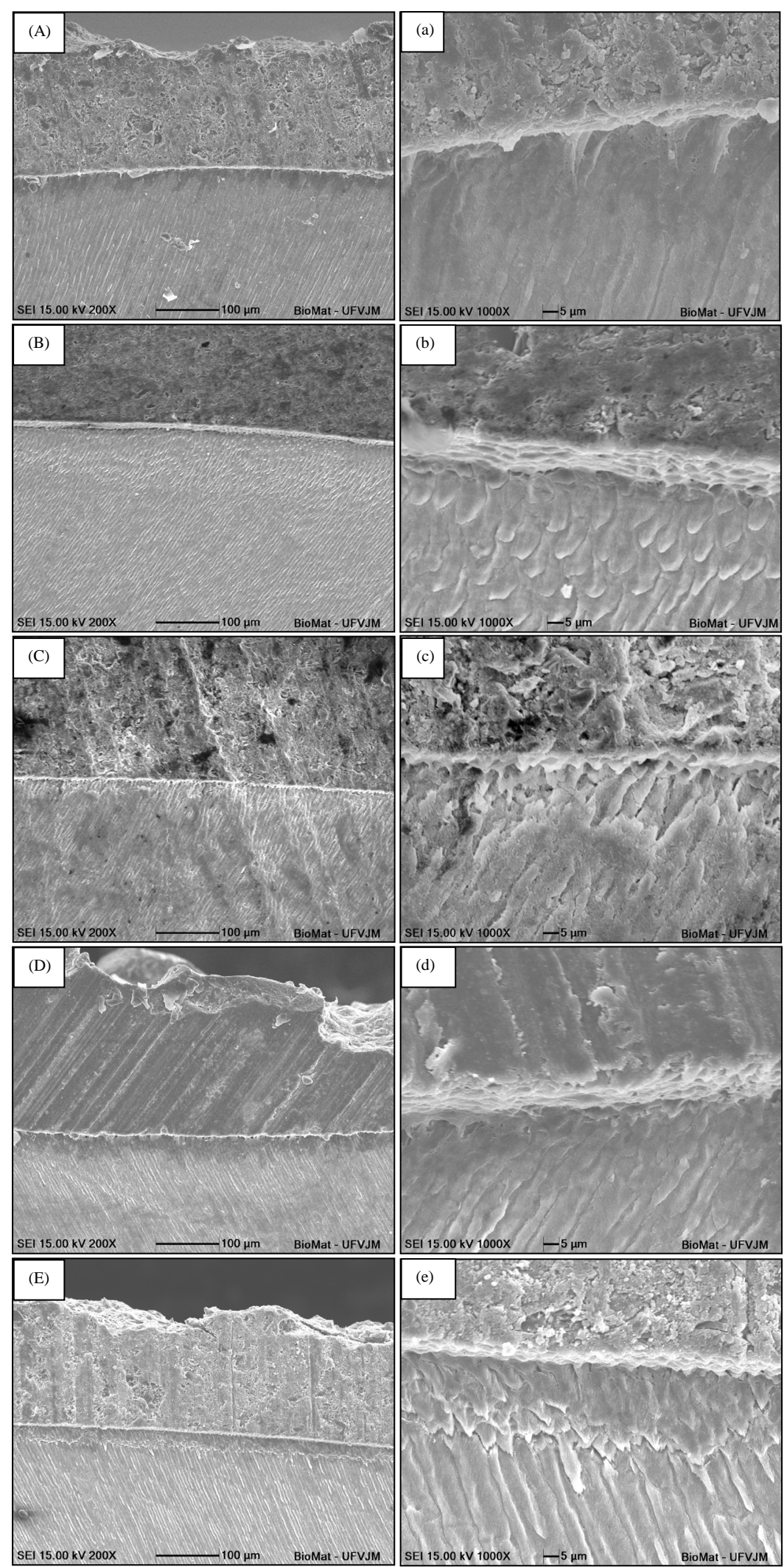

Figure 1. SEM photomicrographs of resin/enamel interfaces at 200× and 1000× magnifications: G1-CO ((A) and (a)), G2-SB ((B) and (b)) and G3-FMO ((C) and (c)), G4-TXT ((D) and (d)) and G5-TP ((E) and (e)). 


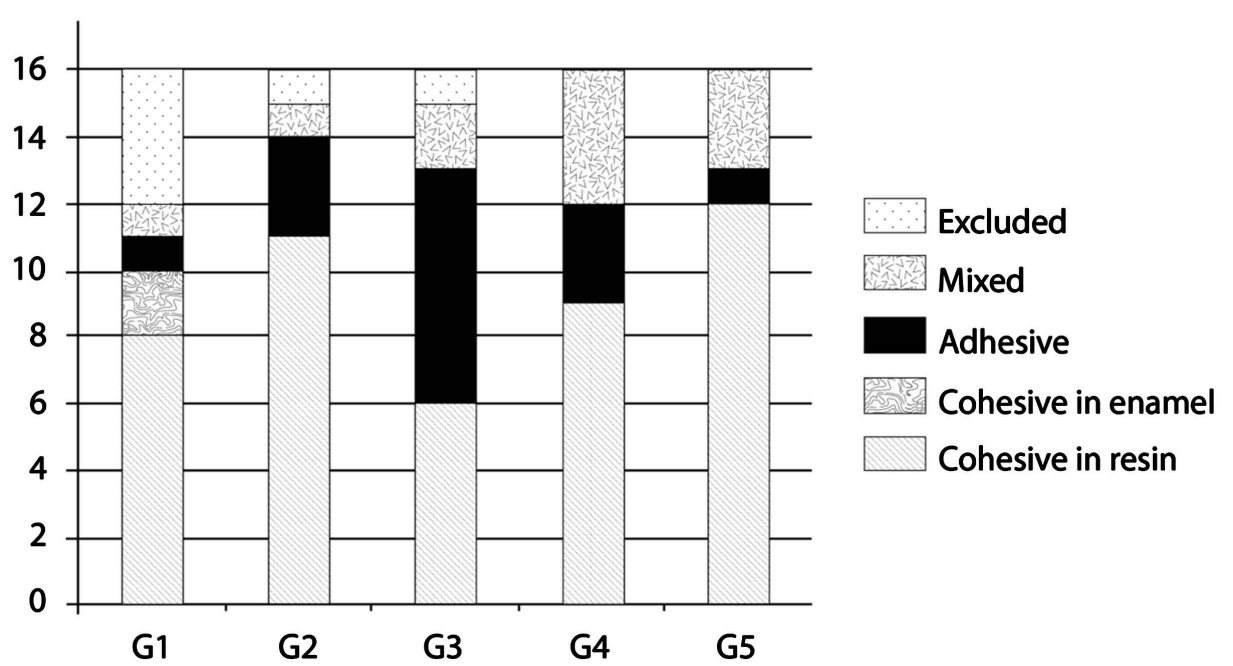

Graph 1. Graph representative of fracture types at enamel/cementing agent interface after the shear bond strength test.

Table 1. Representative table of the values of bond strength to the enamel of the materials used for bonding brackets.

\begin{tabular}{|c|c|c|c|c|}
\hline \multicolumn{5}{|c|}{ Bond strength (MPa) } \\
\hline Group & Mean & SD & $p^{*}$ & Post-hoc ${ }^{* *}$ \\
\hline G1 & 22.25 & 10.71 & & A \\
\hline G2 & 14.85 & 5.17 & & $\mathrm{AB}$ \\
\hline G3 & 11.63 & 7.7 & 0.010 & B \\
\hline G4 & 15.12 & 8.3 & & $\mathrm{AB}$ \\
\hline G5 & 14.92 & 7.68 & & $\mathrm{AB}$ \\
\hline
\end{tabular}

${ }^{*}$ ANOVA. ${ }^{* *}$ test t-student $(p<0.05)$.

with high intensity peaks of Si, in addition to carbon (C) and oxygen (O). The characteristic peaks of these materials may be observed in the spectrum of G1 (Figure 2(a)). Its spectra in areas of the hybrid layer also showed the same pattern and may be represented by spectrum of G3 (Figure 2(b)). The hybrid layer spectra of G2 (Figure 2(c)) and G5 (Figure 2(d)) presented Si inside the prism of etched enamel.

\section{Discussion}

An orthodontic resin must be capable of retaining the brackets firmly bonded to the teeth throughout treatment, resisting masticatory forces and those generated by orthodontic mechanics [11] [12]. However, it is important for orthodontists to know the properties and technique used with materials, in order to prevent bond eventual failures that may lead to bracket debonding, causing loss of efficiency in tooth movement, in addition to time and economic loss to the orthodontist. The present results show that all resin presented bond strength to enamel above the acceptable values for the use in cases of orthodontic bracket bonding. Also, the current results reinforce the idea of the individuality of clinical cases.

The effects of aging caused by thermal cycling have negative consequences on bond strength repair, in comparison with non-thermal cycled samples, irrespective of the composite and enamel conditioning applied [8] [13]. Davidson et al. [14], examined the durability of the shear bond strength of some adhesive systems after they had been submitted up to 300 thermal cycles and observed a significant reduction in bond strength. Price et al., [15] related that up to 5000 thermal cycles the results showed a negative effect on bond strength. In the present study, 3000 cycles were performed considering the thermal cycling is the way to simulate the changes in humidity and temperature in the oral environment, enabling obtainment a condition closer to the clinical situations. 


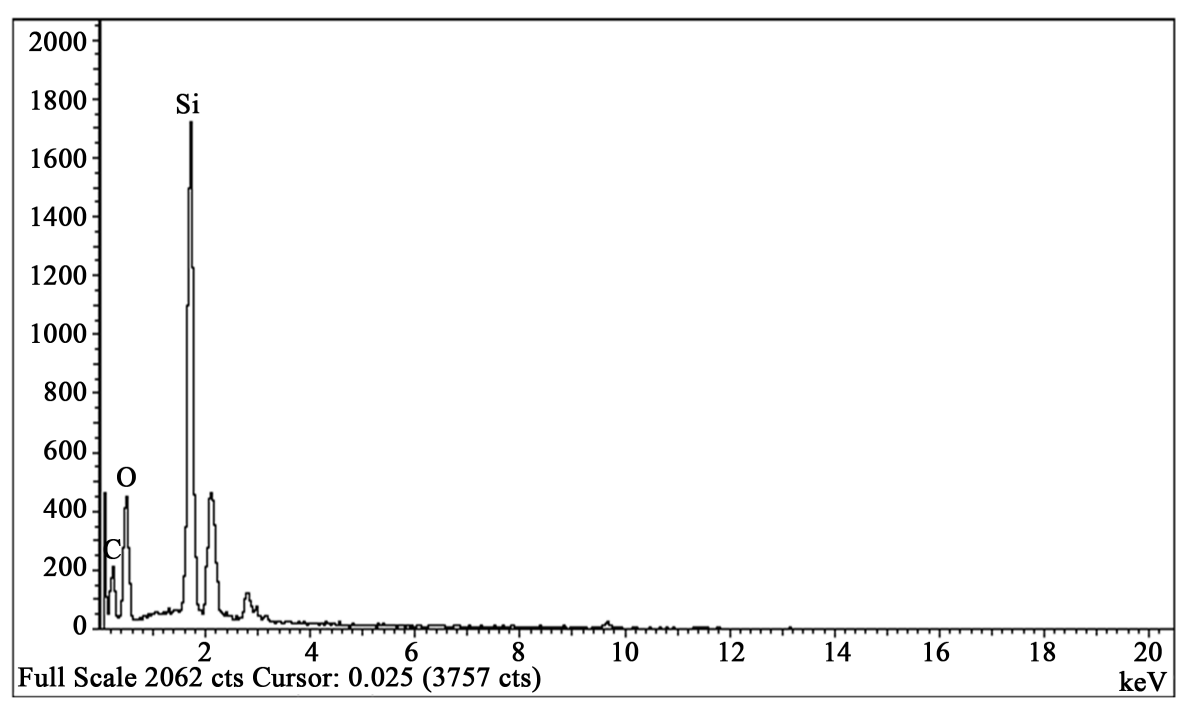

(a)

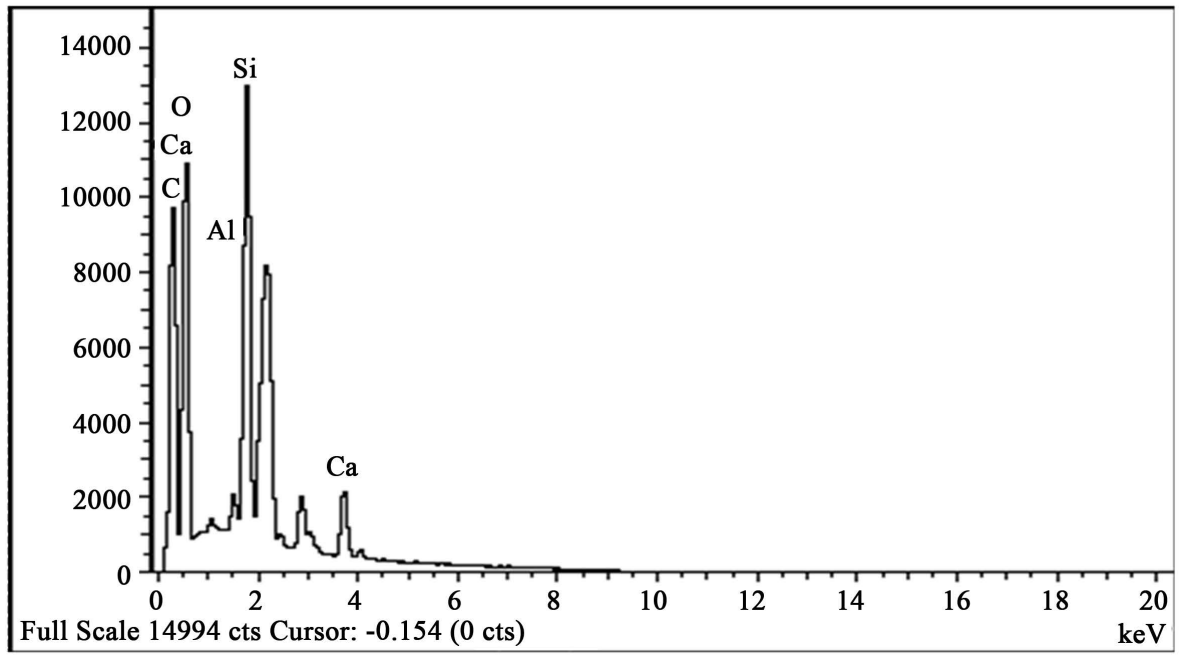

(b)

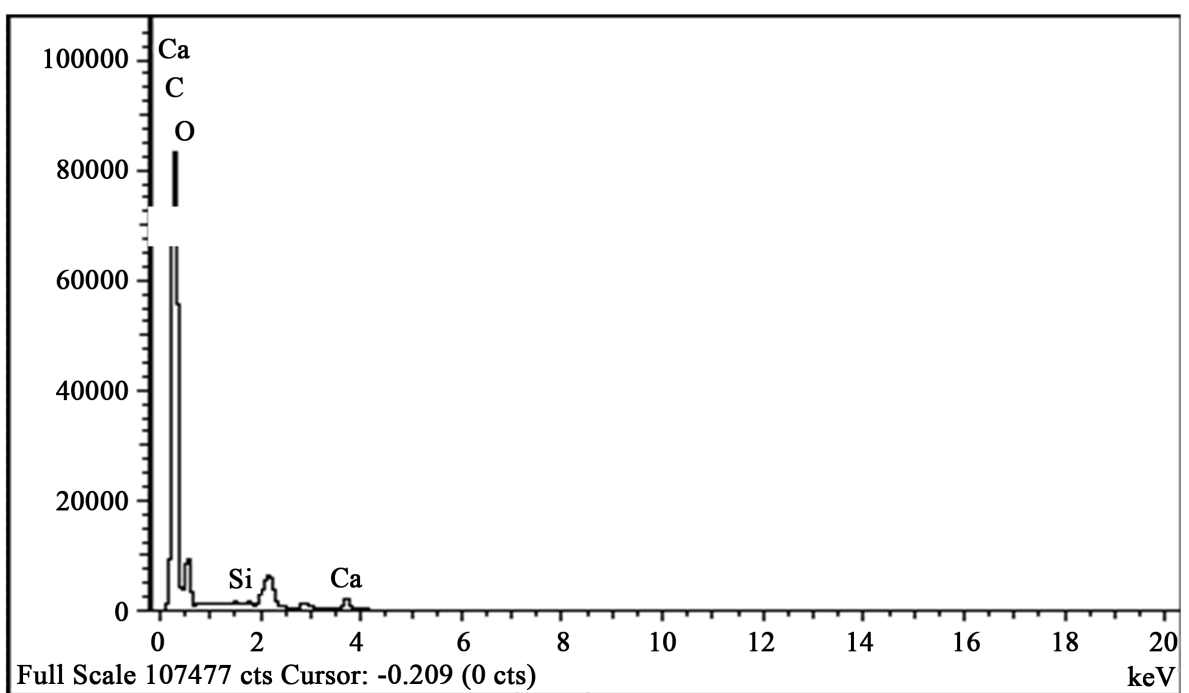

(c) 




(d)

Figure 2. EDS Spectra of G1 - CO (a), showing peaks of Si, C and O, representing the EDS spectra of all the groups; hybrid layer area of G3-FMO (b) presenting peaks of $\mathrm{Ca}, \mathrm{Si}$ and $\mathrm{Al}, \mathrm{C}$ and O; hybrid layer of G2-SB (c) presenting peaks of Ca, Si, C and O; hybrid layer of G5-TP (d) showing peaks of $\mathrm{Si}$.

As well as Evans and Powers [16], also maintained control on bonding to avoid excesses, pressing the brackets against the tooth surface. According to these authors' report, there is a gradual reduction in the bond strength of resins, as the thickness of composites under the devices is increased. Comparing the results of this study with the reference values, it was found that all the adhesive materials presented adequate bond strength for clinical use. The higher values of bond strength to the enamel of the used materials may be attributed to the etching action of phosphoricacid, which offers the advantage of increased bond strength. However, phosphoric acid etching is able to cause a greater degree of enamel loss [11].

The values found in this study were higher than the minimum values, $60 \mathrm{Kgf} / \mathrm{cm}^{2}(5.88 \mathrm{MPa})$ to $80 \mathrm{Kgf} / \mathrm{cm}^{2}$ (7.84 MPa), accepted for the shear bond strength of an adhesive. CO presented significant difference when compared with the other groups, as occurred in the studies of Kawakami et al. [17] (20.10 $\pm 1.44 \mathrm{MPa}-48$ hours and $20.62 \pm 1.53 \mathrm{MPa}-10$ days) and Correr Sobrinho et al. [18] (24.54 $\pm 6.98 \mathrm{MPa})$, who also found higher shear bond strength values when they used Concise resin. Bis-GMA-based resins with a high percentage of particles, such as Concise, presented the best physical properties for bearing the forces produced during mastication and orthodontic movement, in addition to ameliorating unfavorable condition such as humidity found in the oral cavity [19]. In SB the bond strength was higher than it was in FMO, however, lower than it was in the other groups. This may be justified because it is a chemically polymerized material without prior manipulation, as the catalyzer (liquid) is mixed to the base paste only by the pressure exerted at the time of putting the bracket into place. This procedure may lead to incomplete polymerization of some portions of the material, which compromises its bond strength and make it difficult to obtain homogeneity of the results of this bonding system. FMO presented the less expressive result in the shear bond strength tests, a result similar to that of the study of Ianni Filho et al. [20], which showed a value of $11.7 \mathrm{MPa}$ for the same test. The lower values found in this group in the present study may be justified by the absence of primer and because the resin has a low inorganic filler content, as previously reported by Newman et al. [21]. TXT presented a lower bond strength value only in comparison with CO. This may have been due to the presence of a high percentage of silicon filler particles in its organic matrix, in addition to prior acid etching of the enamel. G5 (TP: $14.92 \pm 7.68 \mathrm{MPa}$ ) presented a satisfactory bond strength due to the formation of a completely hybrid bond system that suffered less interference from humidity. This is a cementation system that provides acid etching and primer application all in one procedure, eliminating variables that may harm bonding. Another factor that may have had a direct influence on the bond strength of TP is its lower viscosity. Its fluidity allowed penetration of a larger quantity of material into the micro retentions in the enamel, created by the Transbond Plus Self Etching Primer, allowing increase wetting of the substrate surface, and therefore, bonding. 
After the shear bond strength tests, the predominant type of fracture in CO, SB, TXT and TP was cohesive in resin, corroborating the studies of Penido et al. [22] and Buyukyilmaz et al. [23]. However, in in vivo studies, these authors verified that the bond failure occurred at the cement/bracket interface, and that this type of fracture is frequently found in clinical practice. It is the most desirable type, since facture at this interface may cause damage to the enamel, due to strangulation of the resin that remains between the bracket mesh, making this area more fragile [24]. Two samples from CO presented enamel fracture, a result similar to that found by Penido et al. [25]. Only FMO presented mostly adhesive fractures. Also, there were mixed fractures in all groups that might be the result of mechanical retention of the bracket base or chemical bonding between the enamel and adhesive.

Bond failure at any of the mentioned interfaces has its own advantages and disadvantages. For instance, bond failure at the bracket-adhesive interface is advantageous because it leaves an intact enamel surface; however removing residual adhesive is time consuming and imposes the risk of enamel damage. On the other hand, bond failure at the enamel-adhesive interface leaves less residual adhesive remnants but the risk of enamel surface damage is increased. Bond failure at the enamel-adhesive interface leaves less adhesive remnants on the enamel surface and therefore decreases the risk of enamel damage during adhesive removal but imposes a higher risk of enamel damage during debonding [26]. Because of the higher bond strength of CO composite, the risk of enamel damage during debonding is high as well.

Microstructural analysis by SEM showed the differences between the shape, size and disposition of inorganic particles in all the resin materials. CO and SB had larger particles distributed in the organic matrix, and FMO, TXT and TP had smaller and more homogeneous particles. In addition, penetration of all the resin cementing agents into enamel was observed, with tag and hybrid layer formation, the latter being thicker in SB, followed by TXT and FMO. The hybrid layer was thinner in SB; probably, the high viscosity of the product may have made it difficult for the resin to penetrate into the pores. The hybrid layer obtained in TP was more continuous, thicker and uniform.

However, tags are considered fundamental for retention of the material, and responsible for sealing the surface, with consequent reduction in marginal leakage [27]. For bracket bonding with the use of different bonding agents, tag and hybrid layer formation are not directly related to its retention to the enamel surface [28] [29]. In $\mathrm{CO}$, the etched enamel and use of this composite, together with a liquid resin probably allowed more favorable conditions for resin penetration, resulting in a better bond.

The TP Self Etching Primer system is more conservative when compared with the conventional system with phosphoric acid. Because it is self-etching, this adhesive system has high demineralizing capacity and favors adequate bond strength for orthodontic bracket bonding, presenting similar strength to that obtained with phosphoric acid etching [30] [31]. A uniform and continuous hybrid layer was observed when associated with the TP Color Change adhesive system. In addition to satisfactory bond strength to enamel and minimization of marginal leakage, simplification of the bonding procedure, significant reduction in clinical time, and bracket removal without harm to the enamel surface, appear to be factors making it advantageous to use this association.

\section{Conclusion}

All the groups presented mean values of bond strength to enamel above the acceptable values for the use of orthodontic bracket bonding. The study allows one to affirm that the resins SB, FMO, TXT and TP attained excellent bond strength values. Chemically activated resin composite CO presented higher value of bond strength to enamel than the light activated resin materials FMO, TXT and TP. As regards adhesive systems, there appears to be no difference, because the self-etching system TP demonstrated bond strength similar to that of the conventional bonding systems FMO and TXT.

\section{References}

[1] Correr Sobrinho, L., Correr, G.M., Consani, S., Sinhoreti, M.A.C. and Consani, R.L.X. (2002) Influence of Post-Fixation Time on Shear Bond Strength of Brackets Fixed with Different Bonding Materials. Pesquisa Odontológica Brasileira, 16, 43-49.

[2] Pithon, M.M., Dos Santos, R.L., de Oliveira, M.V., Ruellas, A.C. and Romano, F.L. (2006) Metallic Brackets Bonded with Resin-Reinforced Glass Ionomer Cements under Different Enamel Conditions. Angle Orthodontist, 4, 700-704.

[3] Bengtson, N.G., Bengtson, A.L., Carvalho, D.S. and Rossetto, S.M.A. (2003) Comparative Study of Brackets Bond Strength with for Bonding Agents. Revista Dental Press de Ortodontia e Ortopedia Facial, 8, 43-47. 
[4] Danielle, M.P., Fábio, L.R., Ary, S.P., Lídia, P.M. and Darcy, F.N. (2005) Analysis of the Adhesion Quality of Different Metallic Bracket. Revista Dental Press de Ortodontia e Ortopedia Facial, 10, 88-93.

[5] Melo, T.R.N.B., Maia, A.M.A., Cavalcanti, A.L., Nascimento, A.B.L. and Valença, A.M.G. (2006) Avaliação da resistência ao cisalhamento de adesivos monocomponentes em dentes bovinos. Odontologia Clínico-Científica, 5, 275-280.

[6] Mondelli, A.L. and Feitas, M.R. (2007) Comparative Study of the Shear Bond Strength of the Resin/Bracket Interface, Using Three Different Resin Composites and Three Different Treatments in the Base of the Bracket. Revista Dental Press de Ortodontia e Ortopedia Facial, 12, 111-125. http://dx.doi.org/10.1590/S1415-54192007000300012

[7] Isber, H., Ambrosio, A.R., Carvalho, P.E.G., Valle-Corotti, K.M. and Siqueira, D.F. (2011) Comparative in Vitro Study of the Shear Bond Strength of Brackets Bonded with Restorative and Orthodontic Resins. Brazilian Oral Research, 25, 49-55.

[8] International Standards Organization. ISO Standard 11405:2003: Dental Materials-Testing of Adhesion to Tooth Structure. The Organization, Geneva, 2003.

[9] Fowler, C.S., Swartz, M.L., Moore, B.K. and Rhodes, B.F. (1992) Influence of Selected Variables on Adhesion Testing. Dental Materials, 8, 265-9. http://dx.doi.org/10.1016/0109-5641(92)90097-V

[10] Nakabayashi, M. and Takarada, K. (1992) Effect of HEMA on Bonding to Dentin. Dental Materials, 8, 125-130. http://dx.doi.org/10.1016/0109-5641(92)90067-M

[11] Bishara, S.E., Vonwald, L., Laffoon, J.F. and Jakobsen, J.R. (2000) Effect of Altering the Type of Enamel Conditioner on the Shear Bond Strength of a Resin-Reinforced Glass Ionomer Adhesive. American Journal of Orthodontics and Dentofacial Orthopedics, 118, 288-294. http://dx.doi.org/10.1067/mod.2000.104903

[12] Reynolds, I.R. (1975) A Review of Direct Orthodontic Bonding. British Journal of Orthodontics, 2, 171-180.

[13] Rinastiti, M., Ozcan, M., Siswomihardjo, W. and Busscher, H.J. (2011) Effects of Surface Conditioning on Repair Bond Strengths of Non-Aged and Aged Microhybrid, Nanohybrid, and Nanofilled Composite Resins. Clinical Oral Investigations, 15, 625-633. http://dx.doi.org/10.1007/s00784-010-0426-6

[14] Davidson, C.L., Abdalla, A.I. and De Gee, A.J. (1993) An Investigation into the Quality of Dentin Bonding Systems for Accomplishing a Durable Bond. Journal of Oral Rehabilitation, 20, 291-300. http://dx.doi.org/10.1111/j.1365-2842.1993.tb01611.x

[15] Price, R.B., Derand, T., Andreou, P. and Murphy, D. (2003) The Effect of Two Configuration Factors, Time, and Thermalcycling on Resin to Dentin Bond Strengths. Biomaterials, 24, 1013-1021. http://dx.doi.org/10.1016/S0142-9612(02)00441-6

[16] Evans, L.B. and Powers, J.M. (1985) Factor Affecting in Vitro Bond Strength of No-Mix Orthodontic Cements. American Journal of Orthodontics, 87, 508-512. http://dx.doi.org/10.1016/0002-9416(85)90087-9

[17] Kawakami, R.Y., Pinto, A.S., Gonçalves, J.R., Sakima, M.T. and Gandini, L.G. (2003) In Vitro Evaluation of Debonding Pattern in Bonding Interface of Orthodontic Adhesive Materials to Impacted Teeth Enamel: 48-Hour and 10Day Shear Bond Strengths. Revista Dental Press de Ortodontia e Ortopedia Facial, 8, 43-61.

[18] Correr Sobrinho, L., Consani, S., Sinhoretti, M.A.C., Correr, G.M. and Consani, R.L.X. (2001) Evaluation of Shear Bond Strengths of Bracket Bonding Using Different Materials. Revista ABO Nacional, 9, 157-162.

[19] Beech, D.R. and Jalaly, T. (1981) Clinical and Laboratory Evaluation of Some Orthodontic Direct Bonding Systems. Journal of Dental Research, 60, 972-928. http://dx.doi.org/10.1177/00220345810600061201

[20] Ianni, F.D., Silva, T.B.C., Simplício, A.H.M., Loffredo, L.C.M. and Ribeiro, R.P. (2004) Shear Bond Strength—In Vitro-Evaluation of Different Orthodontics Adhesive. Revista Dental Press de Ortodontia e Ortopedia Facial, 9, 9-48.

[21] Newman, G.V., Snyder, W.H. and Wilson Jr., C.E. (1968) Acrylic Adhesives for Bonding Attachments to Tooth Surfaces. The Angle Orthodontist, 38, 12-18.

[22] Penido, S.M.M.O., et al. (1998) Shear Resistance Evaluation of Recycled Brackets and New Rebonding Brackets. Revista Dental Press de Ortodontia e Ortopedia Facial, 3, 45-51.

[23] Buyukyilmaz, T., Usumez, S. and Karaman, A.I. (2003) Effect of Self-Etching Primers on Bond Strength: Are They Reliable. The Angle Orthodontist, 73, 64-70.

[24] Nkenke, E., Hirschfelder, U., Martus, F. and Eberhard, H. (1997) Evaluation of the Bond Strength of Different Bracket-Bonding Systems to Bovine Enamel. European Journal of Orthodontics, 19, 259-270. http://dx.doi.org/10.1093/ejo/19.3.259

[25] Penido, S.M.M.O., Penido, C.V.S.R., Pinto, A.S., Sakima, T. and Fontana, C.R. (2008) In Vivo and in Vitro Study, with or without Thermocycling, of Shear Bond Strength of Brackets Bonded with Halogen Light. Revista Dental Press de Ortodontia e Ortopedia Facial, 13, 66-76. http://dx.doi.org/10.1590/S1415-54192008000300009

[26] Yassaei, S., Davari, A., Goldani, M. and Kamaei, A. (2014) Comparison of Shear Bond Strength of RMGI and Com- 
posite Resin for Orthodontic Bracket Bonding. Journal of Dentistry, 11, 282-289.

[27] Swift Jr., E.J., Perdigão, J. and Heymann, H.O. (1995) Bonding to Enamel and Dentin: A Brief History and State of the Art. Quintessence International, 26, 95-110.

[28] Fjeld, M. and Ogaard, B. (2006) Scanning Electron Microscopic Evaluation of Enamel Surfaces Exposed to 3 Orthodontic Bonding Systems. American Journal of Orthodontics and Dentofacial Orthopedics, 130, 575-581. http://dx.doi.org/10.1016/j.ajodo.2006.07.002

[29] Montasser, M.A., Drummond, J.L., Roth, J.R., Al-Turki, L. and Evans, C.A. (2008) Rebonding of Orthodontic Brackets. Part II: An XPS and SEM Study. The Angle Orthodontist, 78, 537-544. http://dx.doi.org/10.2319/022707-102.1

[30] Cal-Neto, J.P. and Miguel, J.A. (2006) Scanning Electron Microscopy Evaluation of the Bonding Mechanism of a Self-Etching Primer on Enamel. The Angle Orthodontist, 76, 132-136.

[31] Banks, P. and Thiruvenkatachari, B. (2007) Long-Term Clinical Evaluation of Bracket Failure with a Self-Etching Primer: A Randomized Controlled Trial. Journal of Orthodontics, 34, 243-251. http://dx.doi.org/10.1179/146531207225022293 